\title{
Corrigendum: "Homogenized Spectral Problems for Exactly Solvable Operators: Asymptotics of Polynomial Eigenfunctions"
}

\author{
by
}

Julius BorceA, Rikard BøGVAD and Boris SHAPIRo

\begin{abstract}
Here we provide a correct proof of Proposition 6 of [2]. No other results of the latter paper are affected.

2010 Mathematics Subject Classification: 30C15, 31A35, 34E05.

Keywords: asymptotic root-counting measure, Cauchy transform, homogenized spectral problem, exactly solvable operator.
\end{abstract}

\section{$\S 1$. Necessary results and corrected proof}

To make this note self-contained we briefly recall the basic set-up of [2]. Given a $(k+1)$-tuple of polynomials $\left(Q_{k}(z), Q_{k-1}(z), \ldots, Q_{0}(z)\right)$ with $\operatorname{deg} Q_{i}(z) \leq i$ consider the homogenized spectral pencil of differential operators given by

$$
T_{\lambda}=\sum_{i=0}^{k} Q_{i}(z) \lambda^{k-i} \frac{d^{i}}{d z^{i}} .
$$

Introduce the algebraic curve $\Gamma$ associated with $T_{\lambda}$ and given by the equation

$$
\sum_{i=0}^{k} Q_{i}(z) w^{i}=0,
$$

where the polynomials $Q_{i}(z)=\sum_{j=0}^{i} a_{i, j} z^{j}$ are the same as in (1.1).

The curve $\Gamma$ and its associated pencil $T_{\lambda}$ are called of general type if the following two nondegeneracy requirements are satisfied:

Communicated by M. Kashiwara. Received September 22, 2010.

Julius Borcea unexpectedly passed away on April 8, 2009 at the age of 40 .

R. Bøgvad: Department of Mathematics, Stockholm University, SE-106 91 Stockholm, Sweden; e-mail: rikard@math.su.se

B. Shapiro: Department of Mathematics, Stockholm University, SE-106 91 Stockholm, Sweden; e-mail: shapiro@math.su.se 
(i) $\operatorname{deg} Q_{k}(z)=k$ (i.e., $a_{k, k} \neq 0$ ),

(ii) no two roots of the (characteristic) equation

$$
a_{k, k}+a_{k-1, k-1} t+\ldots+a_{0,0} t^{k}=0
$$

lie on a line through the origin (in particular, 0 is not a root of (1.3)).

The first statement of [2] we need is as follows.

Proposition 1. If the characteristic equation (1.3) has $k$ distinct solutions $\alpha_{1}, \ldots, \alpha_{k}$ and satisfies the above nondegeneracy assumptions (in particular, these imply that $a_{0,0} \neq 0$ and $a_{k, k} \neq 0$ ) then

(i) for all sufficiently large $n$ there exist exactly $k$ distinct eigenvalues $\lambda_{n, j}, j=$ $1, \ldots, k$, such that the associated spectral pencil $T_{\lambda}$ has a polynomial eigenfunction $p_{n, j}(z)$ of degree exactly $n$,

(ii) the eigenvalues $\lambda_{n, j}$ split into $k$ distinct families labeled by the roots of (1.3) such that the eigenvalues in the $j$-th family satisfy

$$
\lim _{n \rightarrow \infty} \frac{\lambda_{n, j}}{n}=\alpha_{j}, \quad j=1, \ldots, k .
$$

The main result of [2] is given below.

Theorem 1. In the notation of Proposition 1, for any pencil $T_{\lambda}$ of general type and every $j=1, \ldots, k$ there exists a subsequence $\left\{n_{i, j}\right\}, i=1,2, \ldots$, such that the limits

$$
\Psi_{j}(z):=\lim _{i \rightarrow \infty} \frac{p_{n_{i, j}}^{\prime}(z)}{\lambda_{n_{i, j}} p_{n_{i, j}}(z)}, \quad j=1, \ldots, k,
$$

exist almost everywhere in $\mathbb{C}$ and are analytic functions in some neighborhood of $\infty$. Each $\Psi_{j}(z)$ satisfies equation (1.2), i.e., $\sum_{i=0}^{k} Q_{i}(z) \Psi_{j}^{i}(z)=0$ almost everywhere in $\mathbb{C}$, and the functions $\Psi_{1}(z), \ldots, \Psi_{k}(z)$ are independent sections of $\Gamma$ considered as a branched covering over $\mathbb{C P}^{1}$ in a sufficiently small neighborhood of $\infty$.

The proof requires Lemma 1 and Proposition 2 below. (The proof of the latter proposition suggested in [2] was erroneous and is corrected below.)

Lemma 1 (cf. Lemma 8 of [1]). Let $\left\{q_{m}(z)\right\}$ be a sequence of polynomials with $\operatorname{deg} q_{m}(z) \rightarrow \infty$ as $m \rightarrow \infty$. Denote by $\mu_{m}$ and $\mu_{m}^{\prime}$ the root-counting measures of $q_{m}(z)$ and $q_{m}^{\prime}(z)$, respectively, and assume that there exists a compact set $K$ containing the supports of all measures $\mu_{m}$ and therefore also the supports of all measures $\mu_{m}^{\prime}$. If $\mu_{m} \rightarrow \mu$ and $\mu_{m}^{\prime} \rightarrow \mu^{\prime}$ as $m \rightarrow \infty$, and $u$ and $u^{\prime}$ are the logarithmic potentials of $\mu$ and $\mu^{\prime}$, respectively, then $u^{\prime} \leq u$ in $\mathbb{C}$ with equality in the unbounded component of $\mathbb{C} \backslash \operatorname{supp}(\mu)$. 
Example 1. Consider the polynomial sequence $\left\{z^{m}-1\right\}$. The measure $\mu$ is then the uniform distribution on the unit circle of total mass 1. Its logarithmic potential $u(z)$ equals $\log |z|$ if $|z| \geq 1$ and 0 in the disk $|z| \leq 1$. On the other hand, the sequence of derivatives is given by $\left\{m z^{m-1}\right\}$ and the corresponding (limiting) logarithmic potential $u^{\prime}(z)$ equals $\log |z|$ in $\mathbb{C} \backslash\{0\}$. Obviously, $u(z)=u^{\prime}(z)$ in $|z| \geq 1$ and $u^{\prime}(z)<u(z)$ in $|z|<1$.

In the notation of Theorem 1 consider the family of eigenpolynomials $\left\{p_{n, j}(z)\right\}$ for some arbitrarily fixed value of the index $j=1, \ldots, k$. Assume that $N_{j}$ is a subsequence of the natural numbers such that

$$
\mu_{j}^{(i)}:=\lim _{n \rightarrow \infty, n \in N_{j}} \mu_{n, j}^{(i)}
$$

exists for $i=0, \ldots, k$, where $\mu_{n, j}^{(i)}$ denotes the root-counting measure of $p_{n, j}^{(i)}(z)$. The existence of such $N_{j}$ follows by Helly's theorem from the existence of a compact set $K$ that contains the support of all $\mu_{n, j}^{(i)}$. Notice that for each $i$ the logarithmic potential $u_{j}^{(i)}$ of $\mu_{j}^{(i)}$ satisfies a.e. the identity

$$
u_{j}^{(i)}(z)-u_{j}^{(0)}(z)=\lim _{n \rightarrow \infty, n \in N_{j}} \frac{1}{n} \log \left|\frac{p_{n, j}^{(i)}(z)}{n(n-1) \ldots(n-i+1) p_{n, j}(z)}\right| .
$$

The next proposition completes the proof of Theorem 1 and also shows the remarkable property that if one considers a sequence of eigenpolynomials for some spectral pencil then the situation $u^{\prime}(z)<u(z)$ seen in Example 1 can never occur. In fact, for the validity of Proposition 2 one only needs two assumptions:

(a) $\operatorname{deg} Q_{k}(z)=k$ (i.e., $a_{k, k} \neq 0$, so that all $\alpha_{j}, j=1, \ldots, k$, are non-zero) and (b) $Q_{0} \neq 0$.

Proposition 2. The measures $\mu_{j}^{(i)}, i=0, \ldots, k$, are all equal and the scalar multiple $\widetilde{\Psi}_{j}=C_{\mu} / \alpha_{j}$ of the Cauchy transform of this common measure $\mu_{j}$ satisfies equation (1.2) almost everywhere.

Proof. For $n \in N_{j}$ one has

$$
\frac{p_{n, j}^{(i+1)}(z)}{(n-i) p_{n, j}^{(i)}(z)} \rightarrow C^{(i+1)}(z):=\int_{\mathbb{C}} \frac{d \mu_{j}^{(i)}(\zeta)}{z-\zeta} \quad \text { as } n \rightarrow \infty
$$

with convergence in $L_{\text {loc }}^{1}$. The well-known property of convergence in $L_{\text {loc }}^{1}$ implies that passing to a subsequence one can assume that the above convergence is actually the pointwise convergence almost everywhere in $\mathbb{C}$. It follows that

$$
\frac{p_{n, j}^{(i)}(z)}{n^{i} p_{n, j}(z)} \rightarrow C^{(1)}(z) \ldots C^{(i)}(z)
$$


pointwise almost everywhere in $\mathbb{C}$. We claim that this limit is non-zero a.e. Granted this, consider

$$
u_{j}^{(k)}(z)-u_{j}^{(0)}(z)=\lim _{n \rightarrow \infty, n \in N_{j}} \frac{1}{n} \log \left|\frac{p_{n, j}^{(k)}(z)}{n(n-1) \ldots(n-k+1) p_{n, j}(z)}\right|=0
$$

almost everywhere in $\mathbb{C}$. On the other hand, $u_{j}^{(0)} \geq u_{j}^{(1)} \geq \cdots \geq u_{j}^{(k)}$ by Lemma 1 . Hence the potentials $u_{j}^{(i)}$ are all equal and the corresponding measures $\mu_{j}^{(i)}=$ $\Delta u_{j}^{(i)} / 2 \pi$ are equal as well.

It remains to settle the above claim. Recall that $p_{n, j}(z)$ satisfies the differential equation $T_{\lambda_{n, j}} p_{n, j}(z)=0$, i.e.,

$$
Q_{k}(z) p_{n, j}^{(k)}(z)+\lambda_{n, j} Q_{k-1}(z) p_{n, j}^{(k-1)}(z)+\cdots+\lambda_{n, j}^{k} Q_{0}(z) p_{n, j}(z)=0 .
$$

Therefore,

$$
Q_{k}(z) \frac{p_{n, j}^{(k)}(z)}{n^{k} p_{n, j}(z)}+\frac{\lambda_{n, j}}{n} Q_{k-1}(z) \frac{p_{n, j}^{(k-1)}(z)}{n^{k-1} p_{n, j}(z)}+\cdots+\frac{\lambda_{n, j}^{k}}{n^{k}} Q_{0}(z)=0 .
$$

Using the asymptotics $\lambda_{n, j} \sim \alpha_{j} n$ and the pointwise convergence a.e. in (1.5) we get

$$
\begin{aligned}
Q_{k}(z) C^{(1)}(z) \ldots C^{(k)}(z)+\alpha_{j} Q_{k-1}(z) C^{(1)}(z) \ldots & C^{(k-1)}(z) \\
& +\cdots+\alpha_{j}^{k} Q_{0}(z)=0 .
\end{aligned}
$$

Using the assumption that $Q_{0} \neq 0 \neq \alpha_{j}$, we conclude that $C^{(1)}(z) \neq 0$ a.e. To prove that $C^{(2)}(z)$ is also non-zero a.e., we consider the differential equation satisfied by $p_{n, j}^{\prime}(z)$,

$$
\begin{aligned}
Q_{k}(z) p_{n, j}^{(k+1)}(z)+\left(Q_{k}^{\prime}(z)+\right. & \left.\lambda_{n, j} Q_{k-1}(z)\right) p_{n, j}^{(k)}(z) \\
& +\cdots+\left(\lambda_{n, j}^{k-1} Q_{1}^{\prime}(z)+\lambda_{n, j}^{k} Q_{0}(z)\right) p_{n, j}^{\prime}(z)=0
\end{aligned}
$$

which is obtained by differentiating (1.6). Repeating the previous analysis we get

$$
\begin{aligned}
& Q_{k}(z) \frac{p_{n, j}^{(k+1)}(z)}{n^{k} p_{n, j}^{\prime}(z)}+\frac{Q_{k}^{\prime}(z)+\lambda_{n, j} Q_{k-1}(z)}{n} \frac{p_{n, j}^{(k)}(z)}{n^{k-1} p_{n, j}^{\prime}(z)} \\
&+\cdots+\frac{\lambda_{n, j}^{k-1} Q_{1}^{\prime}(z)+\lambda_{n, j}^{k} Q_{0}(z)}{n^{k}}=0 .
\end{aligned}
$$

Hence in the limit we obtain

$$
Q_{k}(z) C^{(2)}(z) \ldots C^{(k+1)}(z)+\alpha_{j} Q_{k-1}(z) C^{(2)}(z) \ldots C^{(k)}(z)+\cdots+\alpha_{j}^{k} Q_{0}(z)=0,
$$


which implies that $C^{(2)}(z)$ is non-zero a.e. as well. Similarly, $C^{(i)}(z), i \geq 3$, is non-zero a.e., which proves the claim.

The fact that the multiple $\widetilde{\Psi}_{j}=C_{\mu} / \alpha_{j}$ of the Cauchy transform of this common measure $\mu_{j}$ satisfies equation (1.2) almost everywhere follows by (1.7), since the equality of the measures implies that $C_{\mu}=C^{(1)}=C^{(2)}=\ldots$, and thus

$$
Q_{k}(z)\left(C_{\mu}(z)\right)^{k}+\alpha_{j} Q_{k-1}(z)\left(C_{\mu}(z)\right)^{k-1}+\cdots+\alpha_{j}^{k} Q_{0}(z)=0,
$$

which is equivalent to (1.2).

Note that in Example 1, the polynomials $p_{n}(z):=z^{n}-1$ satisfy the differential equation $z p_{n}^{\prime \prime}(z)-(n-1) p_{n}^{\prime}=0$. They may thus be thought of as eigenpolynomials of the pencil $z \frac{d^{2}}{d z^{2}}-(\lambda-1) \frac{d}{d z}$ corresponding to positive integer values $n$ of $\lambda$. The corresponding homogenized pencil $z \frac{d^{2}}{d z^{2}}-\lambda \frac{d}{d z}$ has $Q_{0}=0$, and so does not satisfy the hypothesis of the proposition.

\section{References}

[1] T. Bergkvist and H. Rullgård, On polynomial eigenfunctions for a class of differential operators, Math. Res. Lett. 9 (2002), 153-171. Zbl 1016.34083 MR 1909635

[2] J. Borcea, R. Bøgvad and B. Shapiro, Homogenized spectral problems for exactly solvable operators: asymptotics of polynomial eigenfunctions, Publ. RIMS Kyoto Univ. 45 (2009), 525-568. Zbl 1182.30008 MR 2510511 